Table 1. Course of Disease parameters evaluated in the 19 patients who maintained abatacept or tocilizumab SC injections in the Rheumatology department of Cochin Hospital

\begin{tabular}{lccc}
\hline Parameter, mean(SD) & $\begin{array}{c}\text { Inclusion visit } \\
\text { Switch to SC } \\
(\mathrm{n}=19)\end{array}$ & 6 month visit $(\mathrm{n}=19)$ & $P$-value \\
\hline DAS28 & $2.3(1.2)$ & $2.3(0.7)$ & 0.62 \\
Tender joint count & $2.5(3.5)$ & $1.3(1.7)$ & 0.49 \\
Swollen joint count & $1.3(2.5)$ & $0.9(0.6)$ & 0.35 \\
Patient Global Health (cm) & $3.2(1.9)$ & $2.6(1.8)$ & 0.60 \\
CRP $(\mathrm{mg} / \mathrm{L})$ & $3.2(4.1)$ & $5.2(4.9)$ & 0.56
\end{tabular}

CRP: C-Reactive Protein, SD: Standard Deviation

The combined analysis of these two populations included 41 patients ( 33 rheumatoid arthritis, RA, 7 juvenile idiopathic arthritis, JIA and 1 polymyalgia rheumatica) who switched to SC ABT or TCZ. 26/41 (63.5\%) patients maintained SC injections and IV was re-established in 15/41 (36.5\%). Reasons for returning to IV were poor tolerance of SC injections $(n=6,40 \%)$, worsening symptoms $(n=11$, $73 \%)$, patient preference to see a rheumatologist in hospital $(n=10,67 \%)$ and the high number of SC injections $(n=2,13 \%)$. The proportion of patients returning to IV was higher in RA patients compared to patients with JIA (42\% vs. 14\%, p = 0.08 ). Age and disease duration were not significantly different between patients who maintained SC injections and those who returned to IV (respectively $p=0.97$ and $\mathrm{p}=0.63$ )

Conclusion: Our study suggests that switching from IV ABT or TCZ to SC is an acceptable procedure during the COVID-19 pandemic, especially for patients with JIA.

Acknowledgements: Association AFP-RIC (Angélique Hochedé, Cyrielle Beller, Sandrine Rollot) and the members of the association for their help in the conduction of the survey

Disclosure of Interests: None declared.

DOI: 10.1136/annrheumdis-2021-eular.1355

\section{AB0666 PROGNOSTIC VALUE OF SERUM KREBS VON DEN LUNGEN-6 GLYCOPROTEIN CIRCULATING LEVELS IN COVID-19 PNEUMONIA: A PROSPECTIVE COHORT STUDY}

D. Lobo Prat ${ }^{1}$, I. Castellví ${ }^{1}$, D. Castillo², S. Orozco ${ }^{2}$, A. Mariscal ${ }^{3}$, L. MartínezMartínez ${ }^{3}$, A. M. Millán Arciniegas' ${ }^{1}$, P. Moya ${ }^{1}$, A. Laiz' ${ }^{1}$, C. Díaz-Torné ${ }^{1}$, B. Magallares ${ }^{1}$, S. P. Fernandez-Sanchez ${ }^{1}$, S. Jeria Navarro ${ }^{1}$, L. Sainz Comas ${ }^{1}$, H. Codes ${ }^{1}$, J. Casademont ${ }^{4}$, P. Domingo ${ }^{5}$, H. Corominas ${ }^{1} .{ }^{1}$ Hospital de la Santa Creu i Sant Pau, Rheumatology, Barcelona, Spain; ${ }^{2}$ Hospital de la Santa Creu i Sant Pau, Pneumology, Barcelona, Spain; ${ }^{3}$ Hospital de la Santa Creu i Sant Pau, Immunology, Barcelona, Spain; ${ }^{4}$ Hospital de la Santa Creu i Sant Pau, Internal Medicine, Barcelona, Spain; ${ }^{5}$ Hospital de la Santa Creu i Sant Pau, Infectious Diseases, Barcelona, Spain

Background: Currently, there are no biomarkers to predict respiratory worsening in patients with Coronavirus infectious disease, 2019 (COVID- 19) pneumonia. Objectives: We aimed to determine the prognostic value of Krebs von de Lungen-6 circulating serum levels (sKL-6) predicting COVID- 19 evolving trends. Methods: We prospectively analyzed the clinical and laboratory characteristics of 375 COVID- 19 patients with mild lung disease on admission. SKL-6 was obtained in all patients at baseline and compared among patients with respiratory worsening.

Results: $45.1 \%$ of patients developed respiratory worsening during hospitalization. Baseline sKL-6 levels were higher in patients who had respiratory worsening (median [IQR] 303 [209-449] vs. 285.5 [15.8-5724], $\mathrm{P}=0.068$ ). The best sKL-6 cut-off point was $408 \mathrm{U} / \mathrm{mL}$ (area under the curve $0.55 ; 33 \%$ sensitivity, $79 \%$ specificity). Independent predictors of respiratory worsening were sKL-6 serum levels, age $>51$ years, time hospitalized, and dyspnea on admission. Patients with baseline sKL- $6 \geq 408 \mathrm{U} / \mathrm{mL}$ had a $39 \%$ higher risk of developing respiratory aggravation seven days after admission. In patients with serial determinations, sKL-6 was also higher in those who subsequently worsened (median [IQR] 330 [219-460] vs 290.5 [193-396]; $p<0.02$ ).

Conclusion: sKL-6 has a low sensibility to predict respiratory worsening in patients with mild COVID-19 pneumonia. Baseline sKL- $6 \geq 408 \mathrm{U} / \mathrm{mL}$ is associated to a higher risk of respiratory worsening. sKL-6 levels are not useful as a screening tool to stratify patients on admission but further research is needed to investigate if serial determinations of sKL-6 may be of prognostic use. REFERENCES:

[1] Zhou F, Yu T, Du R, Fan G, Liu Y, Liu Z, et al. Clinical course and risk factors for mortality of adult inpatients with COVID-19 in Wuhan, China: a retrospective cohort study. Lancet. 2020;395(10229):1054-62. 5.
[2] Tian W, Jiang W, Yao J, Nicholson CJ, Li RH, Sigurslid HH, et al. Predictors of mortality in hospitalized COVID-19 patients: A systematic review and meta-analysis. J Med Virol. 2020

[3] Wang D, Li R, Wang J, Jiang Q, Gao C, Yang J, et al. Correlation analysis between disease severity and clinical and biochemical characteristics of 143 cases of COVID-19 in Wuhan, China: a descriptive study. BMC Infect Dis. 2020;20(1):519.

Disclosure of Interests: None declared.

DOI: 10.1136/annrheumdis-2021-eular.1359

\begin{tabular}{|l|l|}
\hline AB0667 & A PROSPECTIVE STUDY INTO COVID-19 LIKE \\
SYMPTOMS IN PATIENTS WITH AND WITHOUT \\
IMMUNE MEDIATED INFLAMMATORY DISEASES OR \\
IMMUNOMODULATING DRUGS
\end{tabular}

L. van Ouwerkerk ${ }^{1}$, A. Van der Meulen ${ }^{2}$, M. Ninaber ${ }^{3}$, Y. K. O. Teng ${ }^{4}$,

T. Huizinga ${ }^{1}$, C. Allaart'. ' ${ }^{1}$ Leiden University Medical Center (LUMC), Rheumatology, Leiden, Netherlands; ${ }^{2}$ Leiden University Medical Center (LUMC), Gastorenterology, Leiden, Netherlands; ${ }^{3}$ Leiden University Medical Center (LUMC), Pulmonology, Leiden, Netherlands; ${ }^{4}$ Leiden University Medical Center (LUMC), Nephrology, Leiden, Netherlands

Background: Patients with an immune mediated inflammatory disorder or post solid organ transplantation (IMIDT), are at risk for infectious complications especially if they are treated with immunosuppressive drugs (imeds). There is stil great uncertainty whether these IMIDT patients are more susceptible to COVID19 than controls, and/or should be advised to avoid taking their immunosuppressive treatment.

Objectives: To evaluate whether patients with IMIDT are more at risk for CLS than controls.

Methods: The IENIMINI study is a prospective cohort study in patients with IMIDT and controls (healthy or no IMIDT) who were identified based on the registration database of the Leiden University Medical Center. Over time, participants registered COVID-like symptoms (CLS) as they occurred, and filled in additional questionnaires. Univariate and multivariate regression analyses were done to identify variables associated with having CLS.

Results: Of the 8670 individuals approached, 2110 with IMIDT and 1067 controls agreed to participate. In March and April, 454 (22\%) of IMIDT patients and $242(23 \%)$ of controls recorded to have CLS, mostly mild with a median (IQR) duration of seven (3-14) days in the IMIDT group and six days (4-11) in the control group. Eleven (5\%) of the IMIDT patients with immunosuppressive medication (imed), $6(3 \%)$ of IMIDTs without imed and $2(1 \%)$ of controls were hospitalized with CLS $(p=0.04)$. In May and June, fewer episodes overall were recorded. Being female (OR $1.4595 \% \mathrm{Cl} 1.15 ; 1.82)$, having a lung disease (OR $1.5095 \% \mathrm{Cl} \mathrm{1.20;1.88)}$ and wearing a face mask (OR $1.4295 \% \mathrm{Cl} 1.13-1.77$ ) were independently associated with a higher risk, while higher age $(\mathrm{OR} 0.9695 \% \mathrm{Cl}$ 0.96;0.97) and having an IMIDT with immunosuppressive medication use (OR $0.6895 \% \mathrm{Cl} 0.51 ; 0.91$ ) were independently associated with a lower risk (see Table 1). Similar results were found after data imputation.

Table 1. Univariate \& multivariate analysis of variables associated with having CLS or not (OR with $95 \% \mathrm{Cl}$ )

\begin{tabular}{lccc}
\hline & $\mathrm{n}^{0}$ & Univariate & Multivariate $^{*}$ \\
\hline Sex, female & 2546 & $1.89(1.58 ; 2.25)$ & $1.45(1.15 ; 1.82)$ \\
BMI & 2391 & $0.99(0.97 ; 1.01)$ & $1.00(0.98 ; 1.03)$ \\
Age & 2546 & $0.97(0.96 ; 0.97)$ & $0.96(0.96 ; 0.97)$ \\
IMIDT without imed† & 2546 & $1.00(0.82 ; 1.23)$ & $0.94(0.72 ; 1.24)$ \\
IMIDT with imed $\dagger$ & 2546 & $0.79(0.65 ; 0.97)$ & $0.68(0.51 ; 0.91)$ \\
Smoking (current) & 2463 & $1.35(1.02 ; 1.78)$ & $1.05(0.74 ; 1.50)$ \\
Physical contact with family** & 2220 & $1.47(1.22 ; 1.78)$ & $1.22(0.98 ; 1.53)$ \\
Visiting other people (not family) & 2205 & $1.26(1.05 ; 1.51)$ & $0.96(0.77 ; 1.20)$ \\
Wearing a face mask & 2196 & $1.46(1.20 ; 1.76)$ & $1.42(1.13 ; 1.77)$ \\
Close contact (at work) & 2180 & $1.65(1.34 ; 2.03)$ & $1.27(0.97 ; 1.66)$ \\
Self-reported Diabetes Mellitus & 2381 & $0.69(0.50 ; 0.96)$ & $0.89(0.58 ; 1.36)$ \\
Self-reported lung disease & 2396 & $1.30(1.09 ; 1.54)$ & $1.50(1.20 ; 1.88)$ \\
Self-reported heart disease & 2399 & $0.85(0.69 ; 1.04)$ & $1.09(0.83 ; 1.43)$ \\
Daily alcohol use & 2416 & $0.84(0.71 ; 1.00)$ & $1.20(0.96 ; 1.50)$ \\
Influenza vaccination ${ }^{\star \star *}$ & 2415 & $0.71(0.60 ; 0.84)$ & $0.96(0.76 ; 1.21)$ \\
Solid organ transplantation & 2546 & $0.74(0.54 ; 1.03)$ & $0.79(0.47 ; 1.35)$ \\
Good adherence to lockdown rules & 2245 & $1.17(0.41 ; 3.29)$ & $2.46(0.65 ; 9.38)$ \\
Use of oral corticosteroids & 2546 & $0.84(0.66 ; 1.06)$ & $1.44(0.95 ; 2.20)$ \\
Working outside the house & 2435 & $1.39(1.16 ; 1.68)$ & $0.92(0.71 ; 1.20)$ \\
\end{tabular}

Abbreviations: $B M I=b o d y$ mass index; $C l=$ confidence intervals; $C L S=C o v i d$ like symptoms; $I M I D T=$ with immune mediated inflammatory disorders or transplant organ; no=number of observations; OR=odds ratio.

number of observations: 1835

** physical contact specified as 'holding/shaking hands, hugging etcetera'

*** in autumn $2019+$ control group $=$ reference group 
Conclusion: Between March and July 2020, IMIDT patients, whether or not taking imeds, did not show an increased risk of reported COVID-like symptoms compared to controls. Continuing immunosuppressant drugs as long as not ill, while following the Dutch COVID rules, appears to be safe.

Disclosure of Interests: Lotte van Ouwerkerk: None declared., Andrea van der Meulen Speakers bureau: Dr. van der Meulen reports personal fees from Janssen, grants and personal fees from Takeda, personal fees from Galapogos, grants from Nestle, grants from Norgine, outside the submitted work., Grant/ research support from: Dr. van der Meulen reports personal fees from Janssen, grants and personal fees from Takeda, personal fees from Galapogos, grants from Nestle, grants from Norgine, outside the submitted work., Maarten Ninaber: None declared., Y.K. Onno Teng: None declared., Thomas Huizinga: None declared., Cornelia Allaart: None declared. DOI: 10.1136/annrheumdis-2021-eular.1387

\section{AB0668 $\quad$ THE ONSET OF RHEUMATOID ARTHRITIS AFTER COVID-19 - COINCIDENCE OR CONNECTED?}

V. Derksen ${ }^{1}$, T. Kissel ${ }^{1}$, F. Lamers-Karnebeek ${ }^{2}$, A. Van der Bijl $\left.\right|^{3}$, A. C. Venhuizen ${ }^{4}$, T. Huizinga ${ }^{1}$, R. Toes ${ }^{1}$, A. H. E. Roukens ${ }^{5}$, D. Van der Woude ${ }^{1}{ }^{1}$ Leiden University Medical Center (LUMC), Rheumatology, Leiden, Netherlands; ${ }^{2}$ Bernhoven hospital, Rheumatology, Uden, Netherlands; ${ }^{3}$ Isala hospital, Rheumatology, Zwolle, Netherlands; ${ }^{4}$ Rivierenland Hospital, Rheumatology, Tiel, Netherlands; ${ }^{5}$ Leiden University Medical Center (LUMC), Infectious Diseases, Leiden, Netherlands

Background: COVID-19, caused by severe acute respiratory syndrome coronavirus 2 (SARS-CoV-2), has been suggested to induce autoimmune phenomena. Multiple studies have reported the presence of autoantibodies in patients with COVID-19. Also the presence of anti-citrullinated protein antibodies (ACPA) and flaring of rheumatoid arthritis (RA) after COVID-19 has been described.[1, 2] Furthermore, in rheumatology clinics patients may present with polyarthritis compatible with RA shortly after SARS-CoV-2 infection. However, it is unclear how often ACPA occur after COVID-19 and whether preceding COVID-19 impacts on disease presentation of RA and phenotype of the ACPA response.

Objectives: This study aims to determine the seroprevalence of ACPA after COVID-19 and to investigate the association between preceding COVID-19 infection and disease presentation of new-onset RA, including clinical phenotype and autoantibody response.

Methods: To estimate the prevalence of ACPA after COVID-19 we measured ACPA IgG in samples from 61 patients visiting the specialized post-COVID outpatient clinic of the LUMC 5 weeks after hospitalization, using routine tests or in-house ELISA. Furthermore, we identified 5 patients presenting with polyarthritis compatible with RA after SARS-CoV-2 infection. To study the impact of COVID-19 on disease presentation, we examined clinical phenotype, autoantibody isotype positivity and ACPA IgG variable domain (V-domain) glycosylation of these patients and compared these features to regular RA patients. Autoantibody isotypes, including rheumatoid factor (RF) $\lg M / \lg A$, anti-CCP2 $\lg$ G/ $\lg M /$ $\operatorname{IgA}$ and anti-carbamylated protein antibodies (anti-CarP) IgG were measured using in-house ELISA's. The percentage of V-domain glycosylation of purified ACPA IgG was measured with UHPLC

Results: None of the 61 post-COVID patients tested positive for ACPA 5 weeks after hospitalization, except two patients previously diagnosed with ACPA-positive RA. Thus, we could not observe an increase in ACPA-positivity shortly after COVID-19. Of the 5 patients who developed polyarthritis compatible with RA after SARS-CoV-2 infection, the average age was 63.6 years and 2/5 were female. 4/5 patients had been hospitalized due to severe COVID-19. On average, joint complaints started 6.6 weeks after infection, although two patients reported symptoms before infection. 4/5 patients fulfilled the ACR 2010 criteria for RA. Three patients (patient $1,4,5$ ) were phenotypically very similar to regular new-onset RA patients. Patient 3 had a history of seronegative RA and had been in DMARD-free remission for 5 years. She flared 6 weeks after SARS-CoV-2 infection. Patient 2 had a remarkably different presentation. He was admitted with a suspected septic polyarthritis or pneumonia with reactive polyarthritis 6 weeks after COVID-19. ACPA level was low positive. The patient died unexpectedly after two days and autopsy revealed dilating myocarditis of unclear underlying cause. No causative pathogen could be identified.

Previous studies have shown that RA-patients are most often either seronegative or triple-positive for RF, ACPA and anti-CarP antibodies. Autoantibody measurements on sera of the post-COVID polyarthritis patients revealed a similar pattern (Figure $1 \mathrm{~A}$ ) with two patients being completely seronegative, and three patients positive for a range of autoantibodies. In all post-COVID samples, the percentage of ACPA IgG V-domain glycosylation was significantly increased compared to total IgG (Figure 1B), similar as in regular RA.

Conclusion: In conclusion, we found that the seroprevalence of ACPA is not increased post-COVID and that most patients presenting with polyarthritis after COVID-19 resemble regular RA patients, both regarding clinical phenotype and autoantibody characteristics. Although sample size and follow-up was limited, it appears that RA post-COVID may be coincidence rather than connected.

\section{REFERENCES:}

[1] Vlachoyiannopoulos et al. Ann Rheum Dis, 2020

[2] Perrot et al. The Lancet Rheumatology, 2020.
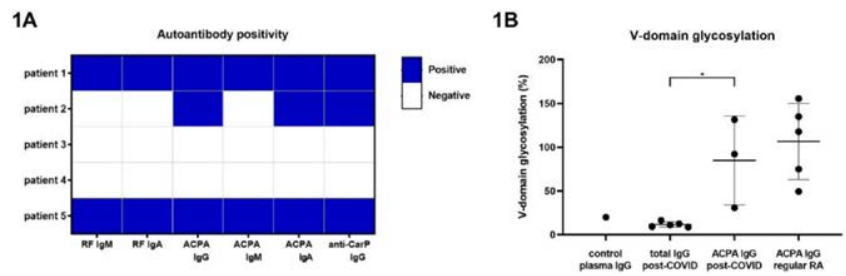

Disclosure of Interests: None declared.

DOI: 10.1136/annrheumdis-2021-eular.1398

\section{$\mathrm{AB0669}$}

DEPRESSION AND ANXIETY MIGHT NOT BE INCREASED DURING COVID-19 PANDEMIC IN PATIENTS WITH AXIAL SPONDYLOARTHRITIS

E. Erpek ${ }^{1}$, D. Solmaz ${ }^{1}$, D. Bayraktar ${ }^{2}$, G. Duran ${ }^{3}$, G. Kabadayi ${ }^{1}$, E. Durak Ediboglu$^{1}$, E. Otman Akat ${ }^{1}$, G. Alp ${ }^{1}$, H. Cinakli ${ }^{1}$, İ. Kurut Aysin ${ }^{1}$, S. Gucenmez ${ }^{1}$, O. Bayindir ${ }^{1}$, M. Ozmen ${ }^{1}$, S. Akar ${ }^{1} .{ }^{1}$ Izmir Katip Celebi University Faculty of Medicine, Rheumatology, Izmir, Turkey; ${ }^{2}$ Izmir Katip Celebi University Faculty of Health Sciences, Department of Physiotherapy and Rehabilitation, Izmir, Turkey; ${ }^{3}$ Dokuz Eylul University, Institute of Health Sciences, Department of Physiotherapy and Rehabilitation, Izmir, Turkey

Background: Anxiety and depression are most common psychiatric disorders in chronic inflammatory rheumatic condition as well as axial spondyloarthritis (axSpA) (1). The prevalence of depression has been reported as $11-64 \%$ depending on the criteria used. Also self-reported depression and anxiety were found to be associated with disease activity and function in $\operatorname{axSpA}(1,2)$. It is observed that mental health is affected among healthy subjects during the COVID-19 pandemic, but this condition has not been systematically reviewed in axSpA patients.

Objectives: We aimed to compare frequency of self-reported depression and anxiety before and during the Covid-19 pandemic in patients with axSpA.

Methods: Seventy-six axSpA patients who were evaluated for the presence of depression and anxiety by using Hospital Anxiety and Depression scale (HADs) before pandemic were included in this study. All participants were classified according to the ASAS axSpA classification criteria. Patients were contacted by phone to participate and complete the HADS questionnaire. Demographic and disease related characteristics including BASDAI, BASFI and Patient Acceptable Symptom State (PASS) were recorded during interview. The HADs cut off value was taken as $>7$ in both groups to define the presence of anxiety or depression. Before and during pandemic period anxiety and depression scores were statistically compared.

Results: The demographic and disease related characteristics of axSpA patients with and without anxiety/depression were summarized in Table 1. The frequency of anxiety (43.4\% vs \%43.4; $p>0.05)$ and depression ( $46.1 \%$ vs $44.7 \%$; $p>0.05)$ were found to be similar before and during pandemic period. Patients with anxiety (HADs >7) and depression (HADs>7) had higher BASDAI and BASFI scores and much less PASS positivity (Table 1). Although the frequency of depression was similar between before and during the pandemic period, symptom severity in depression was slightly increased during the pandemic (Figure 1)

Table 1. Patients' demographics and characteristics according to the presence of anxiety and depression

\begin{tabular}{|c|c|c|c|c|c|c|}
\hline Variables & $\begin{array}{c}\text { Presence of } \\
\text { depression } \\
\text { n:35 }\end{array}$ & $\begin{array}{c}\text { Absence of } \\
\text { depression } \\
n: 41\end{array}$ & $P$ & $\begin{array}{l}\text { Presence of } \\
\text { anxiety n:33 }\end{array}$ & $\begin{array}{l}\text { Absence of } \\
\text { anxiety n:43 }\end{array}$ & $\mathrm{P}$ \\
\hline $\begin{array}{l}\text { Age (years) mean } \\
\quad \pm \mathrm{SD}\end{array}$ & $41.8 \pm 11.2$ & $44.1 \pm 9.3$ & $>0.05$ & $42.0 \pm 10.9$ & $43.6 \pm 10.0$ & $>0.05$ \\
\hline Male $\mathrm{n}(\%)$ & $21(60.0)$ & $26(63.4)$ & $>0.05$ & $18(54.5)$ & $29(67.4)$ & $>0.05$ \\
\hline $\begin{array}{l}\text { Education time (years) } \\
\text { mean } \pm \mathrm{SD}\end{array}$ & $9.6 \pm 4.8$ & $11.0 \pm 4.2$ & $>0.05$ & $9.7 \pm 5.0$ & $10.6 \pm 4.1$ & $>0.05$ \\
\hline Current smoker n(\%) & $18(51.4)$ & $15(36.6)$ & $>0.05$ & $15(45.5)$ & 18(41.9) & $>0.05$ \\
\hline $\begin{array}{l}\text { Alcohol consumption } \\
\mathrm{n}(\%)\end{array}$ & 12(34.3) & 12(29.3) & $>0.05$ & $10(30.3)$ & $14(32.6)$ & $>0.05$ \\
\hline $\begin{array}{l}\text { Current BMI kg/m² } \\
\text { mean } \pm \mathrm{SD}\end{array}$ & $26.0 \pm 4.8$ & $26.8 \pm 4.5$ & $>0.05$ & $26.4 \pm 5.0$ & $26.5 \pm 4.3$ & $>0.05$ \\
\hline $\begin{array}{l}\text { Sleep time (hours) } \\
\text { mean } \pm \text { SD }\end{array}$ & $7.6 \pm 1.7$ & $7.6 \pm 1.3$ & $>0.05$ & $7.5 \pm 1.6$ & $7.7 \pm 1.4$ & $>0.05$ \\
\hline $\begin{array}{l}\text { Current BASDAI } \\
\text { mean } \pm \text { SD }\end{array}$ & $2.5 \pm 1.6$ & $1.4 \pm 1.6$ & $<0.05$ & $2.7 \pm 1.8$ & $1.3 \pm 1.3$ & $<0.001$ \\
\hline $\begin{array}{l}\text { Current BASFI mean } \\
\quad \pm \text { SD }\end{array}$ & $2.4 \pm 2.1$ & $1.1 \pm 1.3$ & $<0.05$ & $2.4 \pm 2.0$ & $1.2 \pm 1.4$ & $<0.05$ \\
\hline PASS positivity n(\%) & $16(45.7)$ & $29(70.7)$ & $<0.05$ & $14(42.4)$ & $31(72.1)$ & $<0.05$ \\
\hline
\end{tabular}

